\title{
COMPLEMENTATION AND RECOMBINATION BETWEEN INDOLE UTILISING TRYPTOPHAN-3 MUTANTS OF NEUROSPORA CRASSA
}

\author{
MAJEED AHMAD, MD. K. U. CHOUDHURY and S. M. ISLAM \\ Department of Botany, University of Dacca, Dacca, Pakistan
}

Received 1.vi.69

\section{INTRODUCTION}

INTERALLELIC complementation among tryptophan-3 (tryp-3) mutants of Neurospora crassa has been investigated by Ahmad and Catcheside (1960), Lacy and Bonner (1961) and Lacy (1965). Lacy and Bonner (1961) did not study any indole utilising $(I U)$ mutant while Ahmad and Catcheside (1960) and Lacy (1965) investigated 2 and $3 I U$ mutants respectively.

Recombinational studies on $I U$ mutants of this locus have been undertaken with 2 primary and 5 secondary mutants by Suyama, Lacy and Bonner (1964), with 1 primary and I secondary by Kaplan, Suyama and Bonner (1964), with 3 primary mutants by Lacy (1965) and with 2 primary mutants by Ahmad et al. (1966).

Since very few primary $I U$ mutants belonging to locus tryp-3 have been previously employed in complementation and recombinational studies such a study was undertaken with ten available $I U$ mutants. The present paper constitutes a report on these investigations.

\section{Materials AND methods}

The 10 indole utilising mutants were obtained by irradiating the conidia of strain Emerson $A$ (5297) with U.V. 2 of these mutants (A45 and A78) have been reported before (Ahmad and Catcheside, 1960) while 8 of them are new (A303, A305, A306, A308, A644, A645, A647 and A668). Mutant aromatic (arom) (Y7655) was utilised as a marker to determine the order of the mutant sites within the tryp-3 locus.

Media and methods used in these studies were the same as reported by Ahmad et al. (1964). Heterocaryon tests were made both in solid and in liquid Vogel's minimal medium (V.M.) (Vogel, 1956). Arom was grown by supplementing V.M. with $6 \mathrm{mg}$. of anthranilic acid, $6 \mathrm{mg}$. of tyrosine, and $6 \mathrm{mg}$. of phenyl alanine per $100 \mathrm{ml}$. Spores from the interallelic crosses were plated on V.M. supplemented with arom nutrients (arom medium). Pseudowilds were determined by crossing an experimental wild-type culture derived from a single spore with the parental wild type. Spores from such a cross were plated on V.M. If germinated spores were observed, 10 were isolated and tested. If the germinated spores were mutants, the wild type culture was classified as pseudowild.

\section{Results}

\section{Complementation}

The $10 I U$ mutants were tested in all possible pairwise combinations for complementing ability. The data are presented in the form of a two dimensional matrix in fig. 1. Tests in solid and in liquid V.M. gave usually similar results. In two cases, however, A303+A308 and A644+A668, the 
growth of heterocaryons was weaker in the solid than in the liquid medium. Furthermore, the heterocaryon formed by A45 with A308 was appreciably less vigorous than the heterocaryons formed between the other pair of mutants.

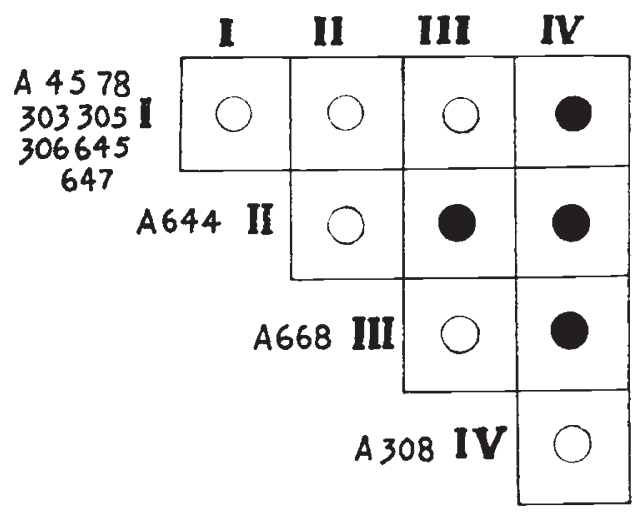

FIG. 1.-Complementation matrix of 10 indole-utilising tryp-3 mutants. Solid circle $=$ complementation present and open circle = complementation absent. Mutants falling under each group are given on the left.

All the mutants complemented at least one other $I U$ mutant. The 10 mutants fell into four groups (fig. l) though mutants within a group failed to form heterocaryons among themselves. Group-I comprised seven mutants while the remaining 3 groups contained one mutant each.

A one dimensional complementation map was made from the above data (fig. 2) assuming that mutants having common defects do not complement.

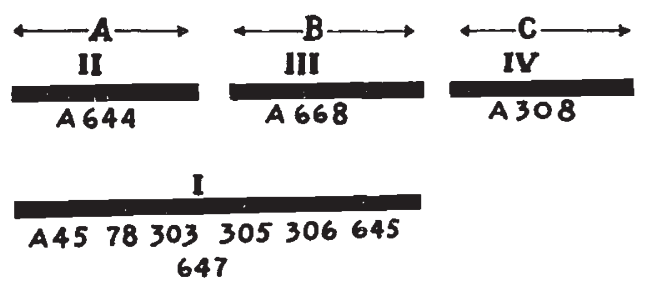

Fig. 2.-Complementation map of the 10 indole-utilising tryp-3 mutants. Letters A, B, and C at the top of the figure indicate complementation units (complons). Functionally defective regions in each group of mutants have been represented by solid lines. Roman numerals above the solid lines indicate the complementation group while designations of specific mutants falling in each group have been written below the solid lines.

This showed that the $I U$ mutants occupy a region of locus tryp-3 which can be subdivided into at least three functionally distinct subunits or complons $(\mathrm{A}, \mathrm{B}, \mathrm{C})$ arranged in a linear manner. Mutants in groups II, III and IV were each defective in single subunits, while mutants in group I were defective in two subunits. No $I U$ mutant was defective in all the three complons.

The three complementation groups II, III and IV have been arranged in the complementation map in the same order as they occur in the genetic map (fig. 3). 


\section{Recombination}

The tryp-3 locus is located in linkage group II at a distance of about 42.5 centimorgans from the centromere (Barratt et al., 1954) and about 20.4 centimorgans on the right of arom locus (Ahmad et al., 1966).

To determine the order of the $10 \mathrm{IU}$ mutants, two double mutants A78 arom and A45 arom were prepared. The sites occupied by different mutants were ordered by crossing the individual mutants with the double mutant A78 arom. The relative positions of A45 and A78 were further confirmed by making the reciprocal cross A45 arom $\times$ A 78 .

The distances of the mutants from A78 were calculated by determining the percentage of recombinants. The data are recorded in table 1 and a genetic map constructed from them is given in fig. 3. The map distance between A45 and A78 was obtained from a weighted mean since data for these two mutants were available from reciprocal crosses.

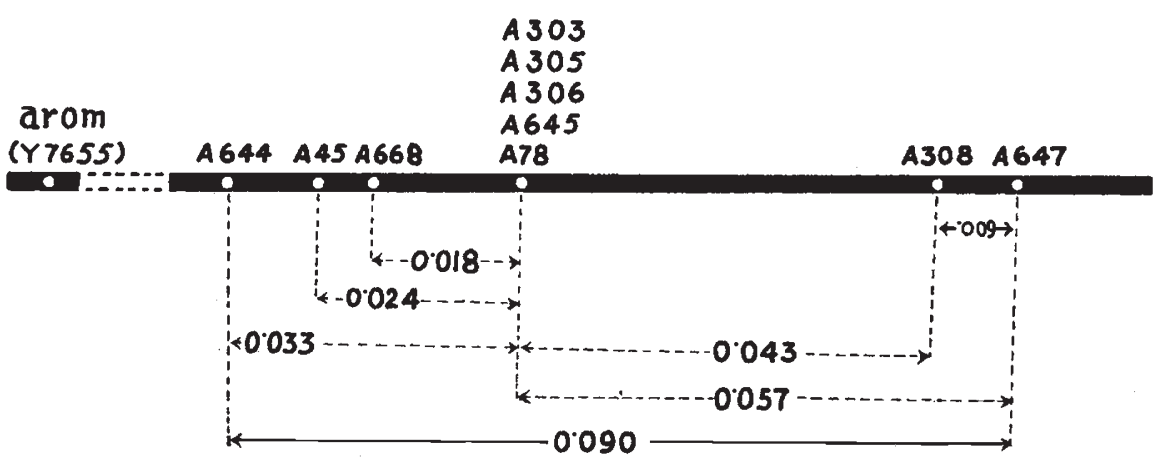

Fig. 3.- Genetic map of indole-utilising region of locus tryp-3. The distances obtained directly from the experimental data have been indicated by dotted lines. The distance obtained by adding individual linear distances between the mutants has been indicated by a continuous line.

It is seen from fig. 3 that the 10 mutants occupy a minimum of 6 mutational sites which are not evenly spaced on the genetical map. Five mutants, A78, A303, A305, A306 and A645, have not been separated by these investigations.

\section{Discussion}

Complementation studies carried on by Ahmad and Catcheside (1960) and Lacy (1965) showed that the indole utilising mutants belonging to locus tryp-3 lacked the capacity to complement one another and occupied only one complon. The present studies have revealed interallelic complementation amongst the 10 mutants tested and have showed that these mutants occupy not one but three complementation units.

A second noteworthy feature is the lack of non-complementing $I U$ mutants. Each one of the 10 mutants complements at least one allelic $I U$ mutant studied. This is in marked contrast to the behaviour of the $\mathcal{N} I U$ mutants, the majority of which do not complement one another (Ahmad and Catcheside, 1960; Lacy and Bonner, 1961).

Recombinational studies on indole utilising mutants have been conducted in the past by Kaplan, Suyama and Bonner (1964), Suyama, Lacy and 
Bonner (1964), Lacy (1965) and Ahmad et al. (1966), who have given the map length occupied by the indole utilisers as $0.020,0.038,0.003$, and 0.022 respectively. The genetic map constructed using 10 indole utilising mutants has given a map length of about 0.09 centimorgan (fig. 3 ). This value is considerably higher than the estimates made by the previous workers because they did not map more than three primary mutants. Furthermore, the map length given by Lacy is not comparable to the other figures because it represents the percentage of true wild type progeny in the total spores plated and not the percentage of recombinants in the viable spores.

The map also shows a clustering of 5 mutants (A78, A303, A305, A306 and A645). This suggests that the frequency of mutations at or near this site is considerably higher than at the other parts of the locus.

By ordering mutants which show the phenomenon of interallelic complementation, complementation maps of loci are obtained (Giles, 1958; Ahmad and Catcheside, 1960). These maps comprise a number of subunits each of which was first designated as complementation unit by Demerec and Hartman (1959). De Serres (1963) " abbreviated the latter term to complon to provide an operational term for the subgroups on a complementation map similar to Benzer's recon, muton, and cistron" (Benzer, 1957). Since it is generally agreed that interallelic complementation follows from proteinprotein interaction (Fincham and Coddington, 1963; Schlesinger and Levinthal, 1963; Garen and Garen, 1963) we can try to give the term complon a functional and physical definition. It is suggested that a segment of polypeptide chain, defects within which cannot be mutually corrected in the multimeric enzyme molecule, may be defined as a complon. It is probable that some or all of these segments may correspond with the individual folds of a polypeptide chain in the formation of the tertiary structure.

\section{Summary}

1. Ten indole utilising tryptophan-3 mutants of Neurospora crassa were tested in all pairwise combinations for complementing ability.

2. Each mutant complemented at least one other mutant.

3. The 10 mutants fell into four groups giving a linear complementation map with three complons.

4. Recombinational studies on the 10 mutants have revealed that they occupy a map length of about 0.09 centimorgan, with five mutants clustered at one point.

5. A functional and physical definition of the term complon has been proposed.

\section{REFERENCES}

AHMAD, M., AND CATCHeside, D. G. 1960. Physiological diversity amongst tryptophan mutants in Neurospora crassa. Heredity, 15, 55-64.

AHMAD, M., KHAlil, MD., KHAN, N. A., AND MOZMADER, A. 1964. Structural and functional complexity at the tryptophan-1 locus in Neurospora crassa. Genetics, 49, 925-933.

AHMAD, M., AHMAD, A., AHMED, B. U., RAHMAN, A., AND RAHMAN, MD., M. 1966. Studies on the organisation of locus tryptophan-3 in Neurospora crassa. Proc. Pak. Natl. Acad. Sci., $3,1-12$.

BARRATT, R. W., NEWMEYER, D., PERKINS, D. D., AND GARNJOBST, L. 1954. Map construction in Neurospora crassa. Adv. Genet., 6, 1-93.

BENZER, s. 1957. The elementary units of heredity. The Chemical Basis of Heredity, 70-93. Ed. by McElroy, W. D. and Glass, B. Johns Hopkins Press, Baltimore. 
demerec, M., and hartman, P. E. 1959. Complex loci in Microorganisms. Ann. Rev. Microbiol., 13, 377-406.

DE SERRES, F. J. 1963. Studies with purple adenine mutants in Neurospora crassa. V. Evidence for allelic complementation among ad-3B mutants. Genetics, 48, 351-360.

FINCHAM, J. R. S., AND CODdington, A. 1963. The Mechanism of Complementation between am mutants of Neurospora crassa. Cold Spring Harb. Symp. Quant. Biol., 28, 517-527.

GAREN, A., AND GAREN, s. 1963. Complementation in vivo between structural mutants of alkaline phosphatase from E. coli. 7. Mol. Biol., 7, 13-22.

grles, N. H. 1958. Mutations at specific loci in Neurospora. Proc. Xth Int. Cong. Gen., 1, 261-279.

KAPlan, S., suYAmA, Y., AND BONNER, D. M. 1964. Fine structure analysis at the td locus of Neurospora crassa. Genetics, 49, 145-158.

LACY, A. M. 1965. Structural and physiological relationships within the td locus in Neurospora crassa. Biochem. Biophys. Res. Comm., 18, 812-823.

LACY, A. M., AND BONNER, D. м. 1961. Complementation between alleles of the td locus in Neurospora crassa. Proc. Natl. Acad. Sci. U.S., 47, 72-77.

SChlesinger, M. J., AND levinthal, c. 1963. Hybrid protein formation of $E$. coli alkaline phosphatase leading to in vitro complementation. f. Mol. Biol., 7, 1-12.

SUYAMA, Y., LACY, A. M., BONNER, D. M. 1964. A genetic map of the td locus of Neurospora crassa. Genetics, 49, 135-144.

voGel, H. J. 1956. A convenient growth medium for Neurospora (Medium N.). Microb. genet. Bull., 13, 42-43.

\title{
SPERM UTILISATION AND RADIATION SENSITIVITY IN DROSOPHILA MELANOGASTER
}

\author{
B. M. SLIZYNSKI \\ Mutagenesis Research Unit of the M.R.C. Institute of Animal Genetics, Edinburgh University
}

Received 10.vi.69

\section{INTRODUCTION}

IN a study of X-ray induced structural changes in Drosophila melanogaster Slizynska (1963) found heterogeneity among males in their response to the treatment. She suggested that this heterogeneity may be due to the differences in the proportion of spermatogonia that were in a sensitive stage at the time of irradiation. High sensitivity would thus be caused by a high proportion of sensitive cells. A change in the proportion of cells in any stage of spermatogenesis may affect the number of spermatozoa produced per unit of time. Thus more offspring would be produced per unit of time when cell cycles of spermatogenesis were shortened and vice-versa.

Many experiments on mutagenesis have been carried out using mass cultures or pooling the results from many males; this naturally obscured all differences between males. The present experiment was planned to investigate whether there are any natural differences among males in their production of spermatozoa (measured by the number of offspring) in a limited time of 10 days.

\section{Material AND methods}

One hundred one-day-old Ore-K males were given each a batch of four one-day-old virgin females every other day. There were 5 such batches of females in the course of the experiment, thus each male had an opportunity to fertilise 20 females in the period of 10 days. The offspring of females were 